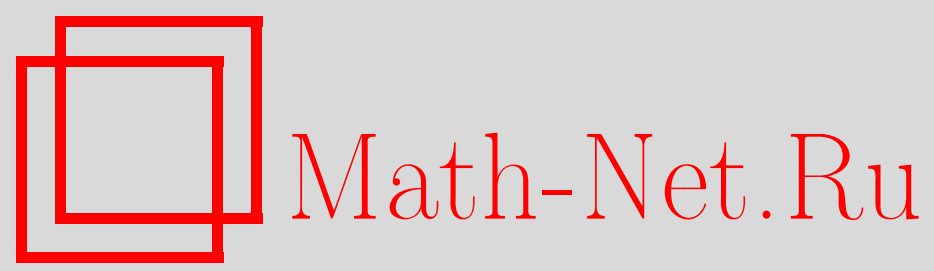

Г. В. Козлов, Л. А. Довбня, И. В. Долбин, Ю. С. Липатов, Взаимодействие основных компонент в дисперсно-наполненных композитах, Вестн. Сам. гос. техн. ун-та. Сер. Физ.-мат. науки, 2002, выпуск 16, 140-144

DOI: https://doi.org/10.14498/vsgtu111

Использование Общероссийского математического портала Math-Net.Ru подразумевает, что вы прочитали и согласны с пользовательским соглашением

http: //www.mathnet.ru/rus/agreement

Параметры загрузки:

IP : 54.157 .27 .8

26 апреля 2023 г., 14:39:52 


\title{
ВЗАИМОДЕЙСТВИЕ ОСНОВНЫХ КОМПОНЕНТ В ДИСПЕРСНО-НАПОЛНЕННЫХ КОМПОЗИТАХ
}

\begin{abstract}
Использование концепции структуры полимерного дисперсно-наполненного композита как совокупности двух фракталов (мультифракталов) совместно с методами фрактального анализа и сеточными моделями позволило получить аналитическое соотношение, определяющее степень «возмущения» структуры полимерной матрииы, и факторы, на нее влияющие. Продемонстрирована важность состояния поверхности частии наполнителя и степени их агрегации. Выполнен предварительный анализ факторов, влияющих на величину фрактальной размерности поверхности агрегатов частии наполнителя.
\end{abstract}

В работе [1] предложено рассматривать структуру дисперсно-наполненного полимерного композита как совокупность двух фракталов (мультифракталов) - каркаса частиц наполнителя и полимерной матрицы. В этой же работе рассмотрено взаимодействие двух указанных фракталов и показано, что оно, в основном, сводится к «возмущению» структуры полимерной матрицы каркасом частиц наполнителя. Основным физическим смыслом термина «возмущение» структуры является снижение в ней степени локального порядка. Определенная в рамках кластерной модели структуры аморфного состояния полимеров [2] указанная степень локального порядка контролирует все важнейшие свойства полимерной матрицы [3-5] и, следовательно, композита [6]. Поэтому представляет интерес выяснение вопроса, какие параметры двух указанных фракталов определяют степень «возмущения» структуры полимерной матрицы, и получение аналитического соотношения для описания этого эффекта, что и является целью настоящей работы. Указанные задачи решались на примере двух серий графитонаполненного полигидроксиэфира (ПГЭ-Гр) [1,7].

Использованы две серии композитов ПГЭ-Гр: с неактивированным (ПГЭ-Гр-І) и активированным смесью серной и азотной кислот (ПГЭ-Гр-ІІ) наполнителями. Подробности получения композитов ПГЭ-Гр приведены в $[1,7]$. Каждая серия включала композиты с объемным содержанием графита $\varphi_{H}=0,0088-0,176$.

Ударные испытания по методике Шарпи образцов с острым надрезом (длина надреза $a=0,22$ мм) композитов ПГЭ-Гр выполнены на маятниковом копре, снабженном пьезоэлектрическим датчиком нагрузки, сигнал с которого непосредственно подавался на запоминающий осциллограф модели С8-13. Такая установка позволяет получить диаграмму нагрузка-время (Рt) и по ней рассчитать модуль упругости $\mathrm{E}$ [8] и напряжение разрушения $\sigma_{p}$ [9]. Все испытания выполнены при температуре 293 К.

Величина критической скорости освобождения энергии деформации $G_{I_{C}}$ рассчитывалась по данным испытаний образцов с надрезом согласно известному соотношению линейной механики разрушения [10]:

$$
G_{I_{C}}=\frac{\pi a \sigma_{p}^{2}}{E} .
$$

Как известно $[11,12]$, мультифрактальные диаграммы обычно строятся в координатах $\alpha-f$, где $\alpha$ - скейлинговый показатель, характеризующий концентрацию сингулярностей; $f$ - размерность сингулярностей $\alpha$. Величина $\alpha$ представляет собой распределение «размерностей процесса», что позволяет связать ее с энергетическими характеристиками этого процесса (например разрушения), а $f$ описывает спектр размерностей для фрактала опоры (например поверхности разрушения). Соотношение между $\alpha$ и $f$ имеет вид [11, 12]

$$
(q-1) D_{q}=q \alpha(q)-f(q)
$$

где $q$ - индекс, $D_{q}$-размерность, зависящая от $q$ (размерность Реньи).

При $q=0 f+2=D_{0}$, где $D_{0}-$ хаусдорфова размерность. Для двух указанных выше фракталов в качестве $D_{0}$ приняты размерности каркаса частиц наполнителя $D_{\kappa}$ и структуры полимерной матрицы $d_{f}$, методы оценки которых подробно описаны в работе [1]. Вторая по величине размерность $D_{l}$ (при $q=1$, информационная размерность) характеризует наибольший субфрактал, который может быть границей всего мультифрактала, поверхностью разрушения и т.д. [12]. Из 
уравнения (2) следует, что при $q=1 \alpha=f$. Р. Meakin [13] получил соотношение, связывающее «меру процесса» $\alpha$ с удельной энергией $G_{I_{C}}$ :

$$
G_{I_{C}}=L_{c p}^{\alpha-2},
$$

где $L_{c p}-$ усредненный размер образца.

На рис. 1 даны диаграммы $\alpha-f$ для обоих мультифракталов, составляющих структуру дисперсно-наполненных полимерных композитов (каркаса частиц наполнителя и полимерной матрицы), где значения $\alpha$ рассчитаны по уравнению (3), а величины $f$ для каркаса частиц наполнителя и полимерной матрицы определены как $\left(D_{k}-2\right) \quad$ и $\quad\left(d_{f}-2\right)$ соответственно, [12]. Как можно видеть из графиков на рис. 1, приведенные зависимости $\alpha-f$ по своему внешнему виду являются частью общей «куполообразной» мультифрактальной диаграммы $\alpha-f$ и представляют собой ее участок, отвечающий хрупкому разрушению. Последнее ожидалось в силу хрупкого разрушения образцов с острым надрезом в ударных испытаниях [7]. Очевидно, что в такой трактовке одному значению $\alpha$ соответствуют две величины $f$ для каркаса частиц наполнителя и полимерной матрицы. Учитывая уравнение (3), можно сказать, что пластичность композита $G_{I_{C}}$ определяют и $D_{\kappa}$, и $d_{f}$. Роль полимерной матрицы в этой ситуации очевидна величина $G_{I_{C}}$ контролируется уровнем

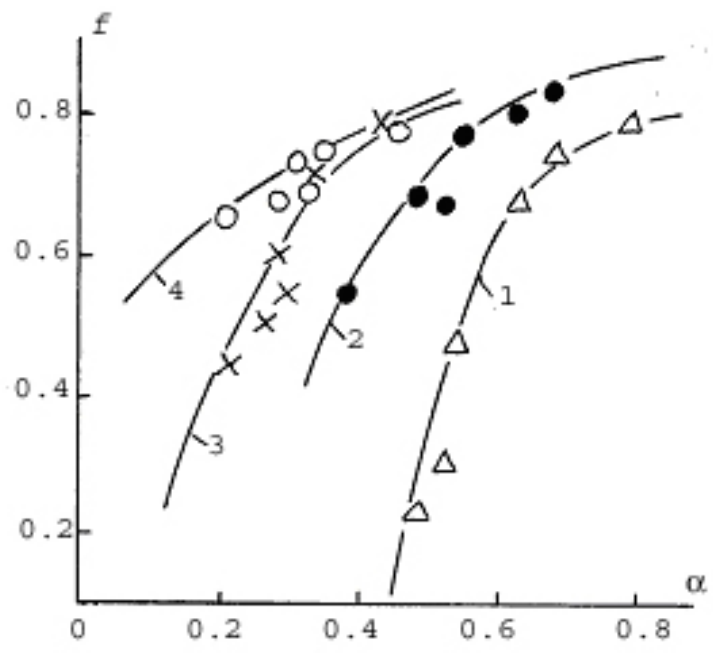

Р и с. 1. Мультифрактальные диаграммы $\alpha-f$ (пояснения в тексте) для каркаса частиц наполнителя $(1,2)$ и полимерной матрицы $(3,4)$ композитов ПГЭ-Гр-I $(1,3)$ и ПГЭ-Гр-II $(2,4)$ локальной пластической деформации, которая реализуется в полимерной матрице композита и зависит от характеристик последней. Роль каркаса частиц наполнителя заключается в видоизменении этих характеристик («возмущении» структуры полимерной матрицы). Следует предположить, что для каркаса частиц наполнителя первым субфракталом с размерностью $D_{l}$ будет поверхность частиц наполнителя, характер которой зависит от степени агрегации исходных частиц наполнителя [14]. Поскольку, как отмечалось выше, $\mathrm{D}_{1}$ соответствует условию $\alpha=f$ и фрактальная размерность поверхности частиц наполнителя $d_{H}=2+f$, то $d_{H}=2+\alpha$. Сравнивая это соотношение с уравнением (3), можно видеть, что пластичность композита определяется величиной $d_{H}$ :

$$
G_{I_{C}}=L_{c p}^{d_{H}-4} .
$$

В свою очередь, пластичность полимерной матрицы определяется ее структурой, а именно, плотностью сетки макромолекулярных зацеплений [15], которую можно трактовать в рамках упомянутой выше кластерной модели [16]. В этом случае величина $G_{I_{C}}$ может быть выражена следующим образом [15]:

$$
G_{I_{C}}=N_{э \phi} E_{a \kappa m},
$$

где $N_{\text {эф }}$ - эффективное число полимерных цепей на поперечное сечение образца, $E_{a к m}-$ энергия активации разрыва макромолекулы.

Величина $N_{э \phi}$ определяется следующим образом [15]:

$$
N_{\ni \phi}=\frac{\sqrt{2 / 3 \pi} \rho l_{c m} N_{A}}{M_{c m} n_{c m}^{1 / 2}},
$$

где $\rho$ - плотность полимера, $l_{c m}$ - длина статистического сегмента, $\mathrm{N}_{\mathrm{A}}$ - число Авогадро, $M_{c m}-$ молекулярная масса статистического сегмента, $n_{c m}$ - число статистических сегментов между узлами зацеплений.

Молекулярная масса $M_{\kappa л}$ участка цепи между кластерами и плотность кластерной сетки $v_{\kappa л}$ связаны между собой так [17]: 


$$
M_{\kappa л}=\frac{\rho N_{A}}{v_{\kappa l}} .
$$

Кроме того, можно использовать два следующих соотношения [18]:

$$
\begin{aligned}
& n_{c m}=\frac{M_{\kappa l}}{M_{c m}} ; \\
& n_{c m}=\frac{2}{S l_{c m} \mathrm{v}_{k r}},
\end{aligned}
$$

где $S$ - площадь поперечного сечения макромолекулы.

Сочетание уравнений (4)-(9) позволяет получить соотношение, описывающее изменение $v_{k z}$ (т.е. степень «возмущения» структуры полимерной матрицы) при введении дисперсного наполнителя:

$$
\mathrm{v}_{\kappa r}=\frac{L_{c p}^{2\left(d_{n}-4\right)} S}{\frac{4}{3} \pi l_{c m} E_{a \kappa m}^{2}} .
$$

Уравнение (10) определяет те характеристики, от которых зависит изменение структуры полимерной матрицы и, следовательно, свойств полимерного композита [6]. Отметим, что величина $v_{\kappa л}$ зависит не только от характеристик наполнителя $\left(d_{H}\right)$, но и молекулярных показателей матричного полимера $\left(S\right.$ и $\left.l_{c m}\right)$. Кроме того, величина $v_{\kappa л}$ зависит (и достаточно сильно) от

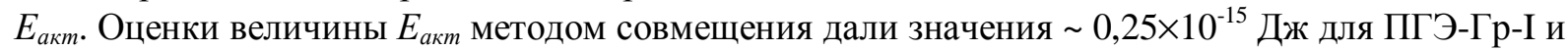
$\sim 0,74 \times 10^{-15}$ Дж для ПГЭ-Гр-ІІ, что на три порядка больше энергии диссоциации связи С-С и на два порядка - значений $E_{a к m}$, учитывающих конформационные изменения цепи при ее деформировании [15]. Понятно, что такое различие обусловлено включением в полученные нами ве-

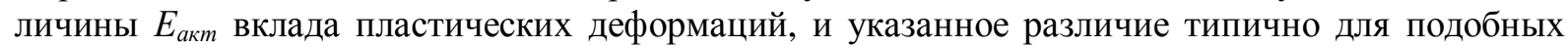
оценок [19]. Необходима дальнейшая работа для выяснения факторов, определяющих величину $E_{\text {aкm }}$ и вида, связывающего эти факторы и $E_{\text {акm }}$ аналитического соотношения.

Величина $L_{c p}$ была оценена как среднее арифметическое размеров образца (длины, ширины и толщины), величина $d_{H}$ рассчитывалась из уравнения (4), а значения $l_{c m}$ получены согласно уравнению [17]

$$
l_{c m}=l_{0} C_{\infty},
$$

где $l_{0}$ - длина скелетной связи основной цепи; $C_{\infty}$ - характеристическое отношение, которое является показателем статистической гибкости цепи [20]. Параметры $S, l_{0}$ и $C_{\infty}$ взяты из литературных источников [20-22].

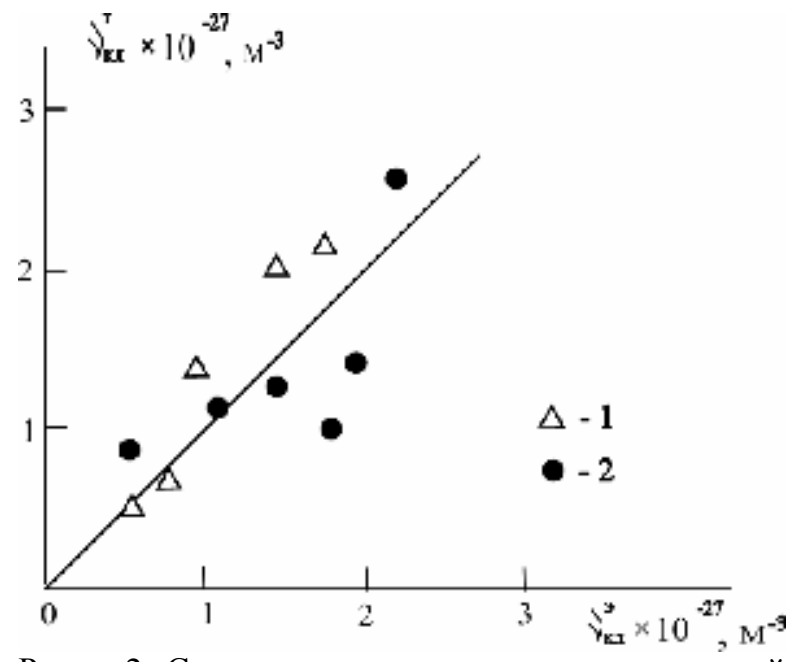

Р и с. 2. Соотношение между экспериментальной $v_{\kappa л}^{\ni}$ и рассчитанной по уравнению (10) $v_{\kappa л}^{T}$ плотностью кластерной сетки зацеплений для композитов ПГЭ-Гр-I (1) и ПГЭ-Гр-II (2) перспективным для целей компьютерного
Сравнение экспериментальных $\mathbf{V}_{\kappa л}^{\ni} \quad$ и рассчитанных по уравнению (10) $v_{\kappa r}^{T}$ величин плотности кластерной сетки зацеплений для обеих серий композитов ПГЭ-Гр приведено на рис. 2. Как можно видеть, получено хорошее соответствие теории и эксперимента, а причины достаточно большого, но симметричного разброса данных $v_{k л}^{T}$ будут рассмотрены ниже.

Уравнение (10) имеет ряд достоинств и недостатков. Укажем некоторые из них. К достоинствам следует отнести точную идентификацию факторов, влияющих на степень «возмущения» структуры полимерной матрицы. После получения упомянутого выше аналитического соотношения для $E_{\text {акm }}$ уравнение (10) не будет содержать эмпирических (подгоночных) коэффициентов, что делает его 
достаткам (скорее, технического характера) следует отнести очень сильную зависимость $v_{k л}^{T}$ от $d_{H}$, что и определило довольно большой разброс на графике (см. рис. 2), а также не очень точную идентификацию параметра $L_{c p}$ в работах $[12,13]$.

Учитывая отмеченную выше важность фрактальной размерности поверхности частиц наполнителя $d_{H}$, рассмотрим факторы, влияющие на ее величину. Как известно $[1,23]$, в композитах ПГЭ-Гр протекает процесс агрегации исходных частиц наполнителя, который можно охарактеризовать двумя параметрами - фрактальной размерностью каркаса частиц наполнителя $D_{\kappa}$, характеризующей их распределение по размерам, и параметром агрегации $K(\rho)$, который служит показателем среднего увеличения размера агрегатов частиц наполнителя (методика определения $K(\rho)$ приведена в работах $[1,24])$. Кроме того, поскольку процесс агрегации усиливается по мере роста $\varphi_{H}[23]$, то и этот показатель может служить одной из характеристик степени агрегации частиц наполнителя. На рис. 3 и 4 приведены зависимости $d_{H}$ от $D_{\kappa}$ и $K(\rho)$ соответственно (зависимость $d_{H}\left(\varphi_{H}\right)$ приведена в работе [23]). Рисунки демонстрируют, что оба указанных фактора воздействуют на величину $d_{t}-$ их увеличение приводит к росту $d_{н}$. Зависимость $d_{H}\left(D_{k}\right)$ линейна и относительно слаба, тогда как при малых $K(\rho)$ происходит значительное увеличение $d_{H}$, но при $K(\rho)$ порядка $3 \times 10^{-2}$ это увеличение существенно замедляется. Экстраполяция графиков $d_{H}\left(\varphi_{H}\right)$ к $\varphi_{H}=0$ дает приближенную меру фрактальной размерности поверхности частиц исходного наполнителя [23], и из этой экстраполяции следует, что активация графита существенно снижает $d_{H}$. Следовательно, наиболее сильно на величину $d_{H}$ влияют фрактальная размерность поверхности частиц исходного наполнителя, а при малых $\varphi_{н}$ (которым соответствуют малые $K(\rho))$ - средний размер частиц. Более слабое влияние оказывают распределение частиц наполнителя по размерам $\left(D_{k}\right)$ и степень наполнения при $\varphi_{k} \geq 0,10$.

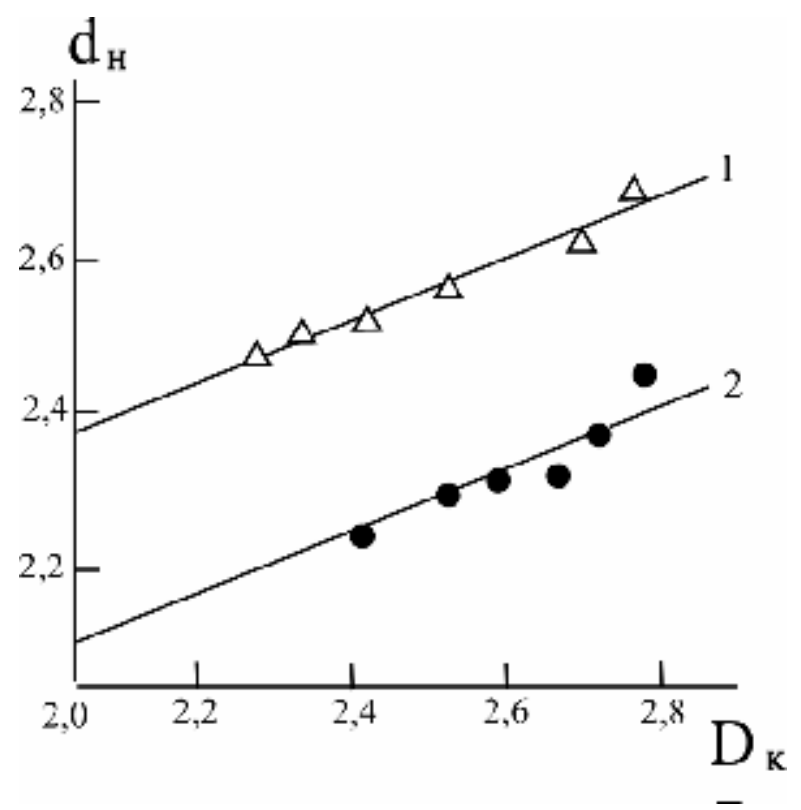

Р и с. 3. Зависимость фрактальной размерности $d_{t}$ поверхности агрегатов частиц наполнителя от фрактальной размерности $D_{\kappa}$ каркаса частиц наполнителя для композитов ПГЭ-Гр-I (1) и ПГЭ-ГрII (2)

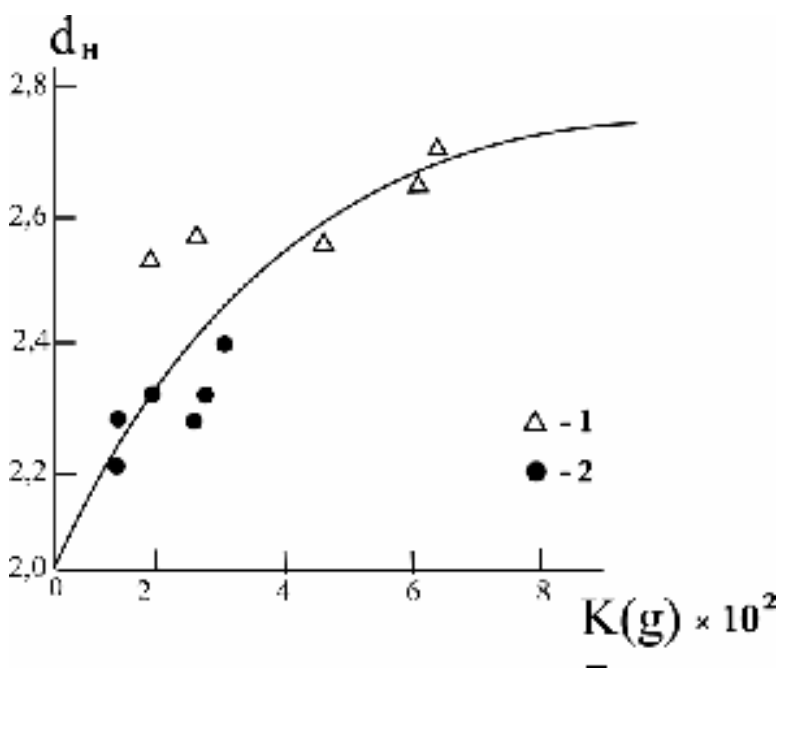

Р и с. 4. Зависимость фрактальной размерности $d_{H}$ поверхности агрегатов частиц наполнителя от параметра агрегации $K(\rho)$ для композитов ПГЭ-Гр-І (1) и ПГЭ-Гр-II (2)

Таким образом, использование концепции структуры полимерного дисперснонаполненного композита как совокупности двух фракталов (мультифракталов) совместно с методами фрактального анализа и сеточными моделями позволило вывести аналитическое соотношение, определяющее степень «возмущения» структуры полимерной матрицы, и факторы, на нее влияющие. Продемонстрирована важность состояния поверхности частиц наполнителя и степени их агрегации. Выполнен предварительный анализ факторов, влияющих на величину фрактальной размерности поверхности агрегатов частиц наполнителя. 


\section{БИБЛИОГРАФИЧЕСКИЙ СПИСОК}

1. Козлов Г.В., Микитаев А.К. Новый подход к фрактальным размерностям структуры полимерных дисперсно-наполненных композитов // Механика композиционных материалов и конструкций. 1996. Т. 2. № 3-4. С. 144-157.

2. Sanditov D.S., Kozlov G.V., Belousov V.N., Lipatov Yu.S. The model of fluctuation free volume and cluster model of amorphous polymers // Ukrain. Polymer J. 1992. V. 1. № 3-4. P. 241-258.

3. Белошенко В.А., Козлов Г.В., Липатов Ю.С. Механизм стеклования сетчатых полимеров // Физика твердого тела. 1994. Т. 36. № 10. С. 2903-2906.

4. Козлов Г.В., Новиков В.У. Физические основы охрупчивания полимерных дисперно-наполненных композитов // Прикладная физика. 1997. № 1. С. 95-101.

5. Новиков В.У., Козлов Г.В. Фрактальная параметризация структуры наполненных полимеров // Механика композитных материалов. 1999. Т. 35. № 3. С. 269-290.

6. Chow T.S. Prediction of stress-strain relationships in polymer composites.// Polymer. 1991. V. 32. № 1. P. 29-33.

7. Козлов Г.В., Абаев А.М., Новиков В.У., Комалов А.С. Свойства наполненного графитом полигидроксиэфира в условиях ударного нагружения // Материаловедение. 1997. № 5. С. 31-35.

8. Козлов Г.В., Шетов Р.А., Микитаев А.К. Методики измерения модуля упругости в ударных испытаниях полимеров.// Высокомолекулярные соединения. А. 1987. Т. 29. № 5. С. 1109-1110.

9. К Козлов Г.В., Шетов Р.А., Микитаев А.К Определение предела вынужденной эластичности при ударном нагружении полимеров по методу Шарпи // Высокомолекулярные соединения. А. 1987. Т. 29. № 9. С. 20122013.

10. Бакнелл К.Б. Ударопрочные пластики. Л.: Химия, 1981. 328 с.

11. Halsey T.C., Jensen M.N., Kadanoff L., Procaccia I., Shraiman B.I. Fractals measures and their singularities: the characterization of strange sets // Phys. Rev. A: 1986. V. 33. № 2. P. 1141-1151.

12. Williford R.E. Multifractal fracture // Scr. Met. 1988. V. 22. № 11. P. 1749-1754.

13. Meakin P. Stress distribution for a rigid fractal embedded in a two-dimensional elastic medium // Phys. Rev. A. 1987. V. 36. № 1. P. 325-331.

14. Avnir D., Farin D., Pfeifer P. Chemistry in noninteger dimensions between two and three. II. Fractal surfaces of adsorbents // J. Chem. Phys. 1983. V. 79. № 7. P. 3566-3571.

15. Mikos A.G., Peppas N.A. Polymer chain entanglements and brille fracture: 2. Autohesion of linear polymers // Polymer. 1989. V. 30. № 1. P. 84-91.

16. Машуков Н.И., Сердюк В.Д., Козлов Г.В., Гладышев Г.П. Оценка напряжения разрушения полиэтилена высокой плотности, модифицированного высокодисперсной смесью $\mathrm{Fe}$ и $\mathrm{FeO}$, в условиях ударного нагружения // Вопросы оборонной техники: 1991. Сер. 15. Вып. 3. № 97. С. 13-15.

17. Козлов Г.В., Новиков В.У. Синергетика и фрактальный анализ сетчатых полимеров. М.: Классика, 1998. $112 \mathrm{c}$.

18. Козлов Г.В., Темираев К.Б., Шетов Р.А., Микитаев А.К. Влияние структурных и молекулярных характеристик на молекулярную подвижность в диблоксополимерах олигоформаль 2,2-ди-(4-оксифенил)-пропанаолигосульфон фенолфталеина // Материаловедение. 1999. № 2. С. 34-39.

19. Берри Дж.П. Разрушение стеклообразных полимеров // Разрушение. Т. 7. Под ред. Г.М. Либовиц. М.: Мир, 1976. C. 7-65.

20. Будтов В.П. Физическая химия растворов полимеров. СПб.: Химия, 1992. 384 с

21. Aharoni S.M. On entanglements of flexible and rodlike polymers // Macromolecules: 1983. V. 16. № 9. P. 17221728.

22. Aharoni S.M. Correlations between chain parameters and failure characteristics of polymers below their glass transition temperature.// Macromolecules. 1985. V. 18. № 12. P. 2624-2630.

23. Козлов Г.В., Овчаренко Е.Н., Липатов Ю.С. Моделирование процессов агрегации частиц наполнителя в полимерных композициях в рамках моделей необратимой агрегации // Докл. НАН Украины. 1999. № 11. С. 128-132.

24. Sumita M., Tsukumo Y., Miyasaka K., Inhikawa K. Tensile yield stress of polypropylene composites filled with ultrafine particles // J. Mater. Sci. 1983. V. 18. № 5. P. 1758-1764. 